\title{
Catabolite Repression and the Induction of Amidase Synthesis by Pseudomonas aeruginosa 8602 in Continuous Culture
}

\author{
By PATRICIA H. CLARKE, MARGARET A. HOULDSWORTH \\ Department of Biochemistry \\ AND M. D. LILLY \\ Biochemical Engineering Section, Department of Chemical Engineering, \\ University College, London
}

(Accepted for publication 25 September 1967)

\begin{abstract}
SUMMARY
Pseudomonas aeruginosa 8602 was grown in continuous culture under steady-state conditions in a carbon-limited medium containing either $20 \mathrm{~mm}$ acetamide or $20 \mathrm{mM}$-acetamide + $10 \mathrm{mM}$-succinate. The amidase specific activity was measured at various dilution rates and found to have a sharp peak at a dilution rate of $0.30-0.35 \mathrm{hr}^{-1}$. Fully constitutive mutants (C I I and L 9) gave curves for amidase activity with the highest values at very low dilution rates $\left(0.05-0.10 \mathrm{hr}^{-1}\right)$ and these decreased as the dilution rate increased. A semi-constitutive mutant ( $\mathrm{C}_{1}$ 7) gave a curve intermediate between that of the wild-type strain and the fully constitutive mutant (C I I). Mutants with decreased sensitivity to catabolite repression by succinate gave curves which declined less steeply at the higher dilution rates. Mutant L 9, a fully constitutive mutant with decreased sensitivity to catabolite repression, had higher specific activities than mutant $\mathrm{C}$ I I at the equivalent dilution rates. Mutant L I I, an inducible mutant with decreased catabolite repressibility, had higher amidase specific activities at high dilution rates than the wildtype inducible strain. It is concluded that in continuous culture under steady-state conditions the specific activity of the inducible amidase of $P$. aeruginosa is determined by the balance between induction and catabolite repression and that catabolite repression is directly related to the growth rate of the culture.
\end{abstract}

\section{INTRODUCTION}

Many inducible enzymes are subject to repression by glucose or other cell metabolites, a process termed catabolite repression by Magasanik (196I). This allows an additional control of the synthesis of such an inducible enzyme, since during growth it will be subject to induction by its substrate and repression by the product or products of catabolic pathways. Almost all the studies on catabolite repression of inducible enzymes have been made in batch culture, usually with exponentially growing bacteria synthesizing the enzyme under conditions of gratuity in the presence of non-substrate inducers.

McFall \& Mandelstam (1963) examined catabolite repression of three inducible enzymes produced by Escherichia coli ( $\beta$-galactosidase, serine deaminase, tryptophanase) in continuous culture, but the results were mainly used to confirm those previously obtained in batch culture. Gorini (1960), with an arginine-requiring auxotroph of $E$. coli strain w, showed that the extent of repression of the synthesis of 
ornithine transcarbamylase by arginine was dependent on the flow rate of the medium. He also found that repression by arginine could be relieved by ornithine and the greater the repression the more ornithine was required to overcome it. Continuous culture experiments on enzyme regulation have been made by Hamlin, $\mathrm{Ng} \&$ Dawes (1967) with Pseudomonas aeruginosa 2F 32. Hamilton \& Dawes (1959) had previously shown that this organism exhibits an unusual diauxie in that when inoculated into glucose + citrate medium the citrate is utilized preferentially to glucose until the citrate concentration has reached a low value. The extent of induction of glucosemetabolizing enzymes in the presence of a constant citrate concentration is directly related to the concentration of exogenous glucose. $\mathrm{Ng} \&$ Dawes (1967) suggest that there is dual control of the phosphorylative enzymes of glucose metabolism with glucose serving as the inducer and citrate as the precursor of the repressing catabolites.

Pseudomonas aeruginosa 8602 is induced to form an aliphatic amidase (acylamide amidohydrolase, EC 3.5.I.4) during growth in the presence of the substrate acetamide or non-substrate inducers, e.g. $N$-acetylacetamide. This enzyme is subject to catabolite repression and it has been found that succinate is a very effective repressor of amidase synthesis by both inducible and constitutive strains growing in pyruvate medium (Brammar, Clarke \& Skinner, 1967). We found previously that when the ingoing medium for a continuous culture growing in a steady state on limiting succinate was changed to succinate + acetamide, the extent of catabolite repression of amidase synthesis was dependent on the dilution rate of the culture (Boddy, Clarke, Houldsworth $\&$ Lilly, 1967). At low dilution rates amidase synthesis started after little or no lag, but at a high dilution rate, $D=0.76 \mathrm{hr}^{-1}$, the lag lasted for more than $4 \mathrm{hr}$ after the change in the ingoing medium. We observed that the amidase specific activities of the cultures when they had reached the new steady state of growth on succinate + acetamide also appeared to be dependent on the dilution rate. Amidase synthesis by $P$. aeruginosa 8602 has now been studied under steady-state conditions at various dilution rates by using the wild-type strain, fully constitutive and semi-constitutive mutants, and mutants with altered susceptibility to catabolite repression.

\section{METHODS}

Organisms. The strains used were Pseudomonas aeruginosa 8602 (wild type) and mutants isolated from it by the techniques described by Brammar et al. (1967). These are listed in Table $\mathbf{I}$. All the strains were made resistant to the lysogenic pseudomonad phage Ps I. The bacteria were maintained on slopes of Lemco agar, subcultured weekly and stored at $4^{\circ}$.

Media. The minimal salts medium contained (g./1.): $\mathrm{K}_{2} \mathrm{HPO}_{4}, \mathrm{I} 2 \cdot 5 ; \mathrm{KH}_{2} \mathrm{PO}_{4}, 3.8$; $\left(\mathrm{NH}_{4}\right)_{2} \mathrm{SO}_{4}, \mathrm{I} \cdot 0 ; \mathrm{MgSO}_{4} \cdot 7 \mathrm{H}_{2} \mathrm{O}, 0 \cdot \mathrm{I}$; trace element solution $5 \mathrm{ml}$. (Kelly \& Clarke, 1962 ). This medium was used to prepare succinate + formamide agar (S/F plates) and succinate +lactamide agar (S/L plates) (Brammar et al. 1967$)$. When required, sodium pyruvate and sodium succinate were added to the medium before autoclaving. Acetamide and $\mathrm{N}$-acetylacetamide solutions were sterilized by membrane filtration and added aseptically to the autoclaved medium.

Continuous culture equipment. An inoculum ( $70 \mathrm{ml}$.) grown on succinate + acetamide minimal salts medium, or an acetamide minimal salts medium, was blown from the seed vessel into a 51 . vessel containing 31 . of the growth medium at a controlled 
temperature of $37^{\circ}$. The $\mathrm{pH}$ value of the medium was controlled at $\mathrm{pH} 7 \cdot 2$ by the addition of $2 \mathrm{M}-\mathrm{HCl}$. Air was supplied through the bottom of the vessel at $\mathrm{I} 1 . / \mathrm{min}$. Growth medium was fed from a 201 . aspirator into the culture vessel by a peristaltic pump. Medium left the vessel by an overflow pipe inserted through the bottom of the vessel. Samples (about $3 \mathrm{ml}$.) were withdrawn through a narrow-bore tube leading to a collecting bottle. Each sample was diluted, part used for the determination of the bacterial concentration, and the remainder cooled rapidly and assayed for amidase within a few minutes.

Dry weight of bacteria. The optical extinctions of diluted bacterial suspensions were measured at $670 \mathrm{~m} \mu$ with a Unicam SP 600 spectrophotometer, and dry weights of bacteria calculated by using the appropriate conversion factor for each dilution rate.

Enzyme assay. Amidase was measured by the hydroxamate method for transferase activity described by Brammar \& Clarke (1964). Except in experiments with $\mathrm{N}$-acetylacetamide as inducer, the sample was diluted several times before assaying $0.1 \mathrm{ml}$. samples at $37^{\circ}$ for $5 \mathrm{~min}$. The amidase specific activity of each sample was calculated as $\mu$ moles acethydroxamate formed $/ \mathrm{mg}$. dry wt bacteria $/ \mathrm{min}$.

Steady-state conditions. The flow rate of the culture medium was adjusted to the required value and the culture allowed to grow for about $24 \mathrm{hr}$ at the new growth rate, to reach steady-state conditions before sampling. Amidase specific activities of bacteria growing under steady-state conditions at each dilution rate were determined by taking the mean of eight consecutive readings made at $15 \mathrm{~min}$. intervals.

\section{RESULTS}

Amidase activities of wild-type bacteria growing under steady-state conditions

Amidase specific activities of cultures of wild-type Pseudomonas aeruginosa 8602 growing in continuous culture were determined at dilution rates from 0.05 to $0.60 \mathrm{hr}^{-1}$. Figure I compares the results obtained with cultures growing on acetamide $(20 \mathrm{~mm})$ as the sole carbon source, with cultures growing on a mixture of acetamide (20 mM)+ succinate (IO mM). In both cases growth was carbon-limited and excess nitrogen was present as ammonium salts. The amidase specific activities of the bacteria increased as the dilution rate was raised, reaching a maximum activity of about $\mathrm{I} 20$ at a dilution rate of $0.30-0.35 \mathrm{hr}^{-1}$. The slopes of the curves were similar at the lower dilution rates for both media. At higher dilution rates the specific activity decreased but in the medium containing succinate + acetamide the amidase specific activity decreased more rapidly until at about $D=0.60 \mathrm{hr}^{-1}$ it was about one-third of that in the medium containing acetamide alone. The values given in Fig. I were obtained in random order; when amidase activities were determined on more than one occasion no difference was detected whether the new dilution rate had been approached from a previously higher or lower dilution rate. The similarity of the two curves suggested that essentially similar control mechanisms were operating in both media, but since the succinate + acetamide medium produced more dramatic changes in amidase activity at slightly lower dilution rates it was decided to use this medium for the experiments with the amidase mutants. 


\section{Amidase activities of constitutive mutants growing in continuous culture}

Pseudomonas aeruginosa 8602 mutant $\mathrm{C}$ II is a magno-constitutive non-inducible strain (Brammar et al. 1967 ). It is as sensitive as the wild type to catabolite repression by succinate (IO mM) in pyruvate medium in batch culture (Table I). Figure 2 shows that when mutant $C_{\text {I I }}$ was grown in continuous culture in the succinate + acetamide medium the highest specific activity was produced at the lowest dilution rate tested $(D=0.06)$ and decreased to a value of $10-15$ at a dilution rate of $0.42 \mathrm{hr}^{-1}$. There was no sharp peak of maximum amidase activity and the curve obtained resembled the falling side of the wild-type curve displaced to a lower dilution rate.

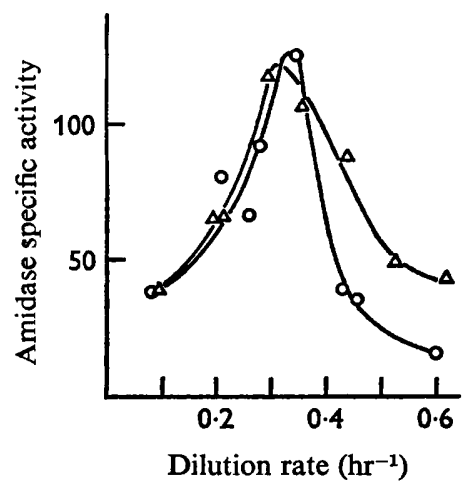

Fig. I

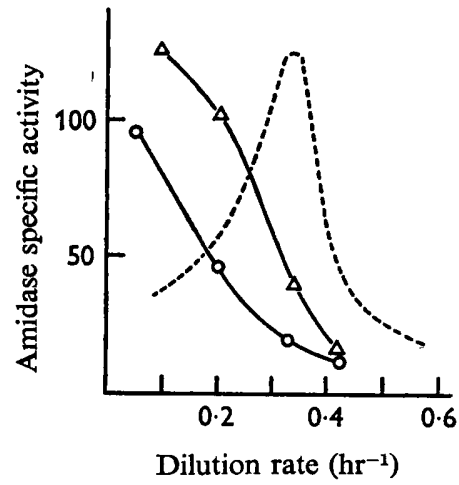

Fig. 2

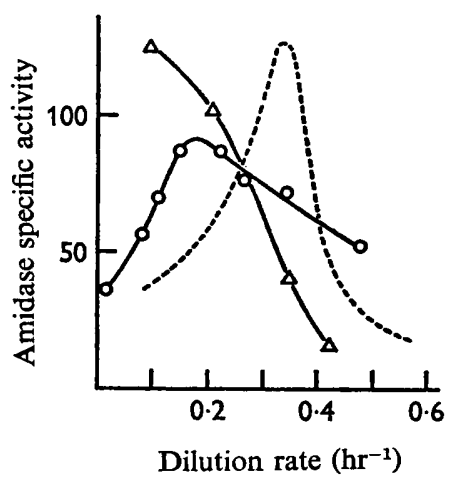

Fig. 3

Fig. I. Pseudomonas aeruginosa $\mathbf{8 6 0 2}$ wild type, grown in continuous culture under steadystate conditions. Amidase activities of cultures grown at various dilution rates. $\mathrm{O}-\mathrm{O}$, Minimal salts medium containing Io mM-succinate $+20 \mathrm{mM}$-acetamide; $\triangle \longrightarrow \triangle$, minimal salts medium containing $20 \mathrm{~mm}$-acetamide.

Fig. 2. The amidase specific activities of fully constitutive mutants of Pseudomonas aeruginosa 8602 grown in continuous culture under steady-state conditions. The minimal salts medium contained $10 \mathrm{~mm}$-succinate +20 mM-acetamide. $\mathrm{O}-\mathrm{O}$, Mutant $\mathrm{C}$ I I ; $\triangle \longrightarrow \triangle$, mutant L 9; - - , wild type on the same medium.

Fig. 3. Mutants with altered catabolite repressibility. Amidase activities of continuous cultures grown under steady state conditions in minimal medium containing $10 \mathrm{mm-succinate}+$ 20 mM-acetamide. $\triangle \longrightarrow \triangle$, Fully constitutive mutant $\mathrm{L}$ 9; $\mathrm{O} \longrightarrow \mathrm{O}$, inducible mutant $\mathrm{L}$ I I ; - - - wild type on the same medium.

Mutant L 9 was isolated in a single step on succinate + lactamide minimal salt agar (S/L plates) after treatment of the wild-type strain with ethylmethane sulphonate. This mutant also grew on succinate + formamide agar (S/F plates) and when tested for rate of enzyme synthesis in batch culture it behaved as a magno-constitutive non-inducible strain. Mutants isolated from S/L plates have been found to be less sensitive to catabolite repression by succinate. The differential rate of amidase synthesis by strain $L 9$ in batch culture in pyruvate medium was almost unaffected by the addition of Io $\mathrm{mm}$ succinate (Table I). In continuous culture the curve obtained for amidase specific activities at various dilution rates resembled that for mutant C I I but was markedly displaced towards the higher dilution rates (Fig. 2). The result was therefore that until 
a dilution rate of about $D=0.40 \mathrm{hr}^{-1}$ was reached, the amidase specific activity of the mutant $\mathrm{L} 9$ cultures was always higher than that of the mutant $\mathrm{C}$ I I culture, for any given dilution rate.

Table I. Properties of mutant strains of Pseudomonas aeruginosa 8602

\begin{tabular}{|c|c|c|c|c|}
\hline Strain & Amidase phenotype & Mutagen* & $\begin{array}{l}\text { Isolation } \\
\text { medium } \dagger\end{array}$ & $\begin{array}{c}\text { Catabolite } \\
\text { repressi- } \\
\text { bility }(\%) \ddagger\end{array}$ \\
\hline Wild type & Inducible & - & - & 98 \\
\hline$C_{\text {II }}$ & Magno-constitutive & spont. & $S / F$ & 85 \\
\hline $\mathrm{C}_{17}$ & Semi-constitutive & u.v. & S/F & 80 \\
\hline$L_{9}$ & Magno-constitutive & ems & $\mathbf{S} / \mathbf{L}$ & 15 \\
\hline L II & Inducible & ems & $\mathbf{S} / \mathbf{L}$ & 60 \\
\hline
\end{tabular}

\section{Amidase activities of an inducible mutant with decreased sensitivity to catabolite repression}

Mutant $\mathrm{L}$ I I was isolated from $\mathrm{S} / \mathrm{L}$ medium after treatment of the wild-type strain with ethylmethane sulphonate. It differed from mutant L 9 in that it grew only slightly better than the wild-type on $\mathrm{S} / \mathrm{F}$ plates and behaved in batch culture as an inducible strain. Table I shows that, when fully induced by $N$-acetylacetamide as non-substrate inducer in pyruvate medium, it was repressed by only $60 \%$ on the addition of $10 \mathrm{~mm}-$ succinate.

Figure 3 compares the results obtained in continuous culture with mutants L II, L 9 and the wild type. It can be seen that the curve for the amidase specific activity of mutant L I I has an upward slope at the lower dilution rates. This had been previously observed with the wild-type inducible strain but not for the fully constitutive mutant $\mathrm{L}$ 9. The amidase specific activity of mutant $\mathrm{L}$ I I was however significantly higher than that for the wild type between dilution rates of 0.05 and $0.20 \mathrm{hr}^{-1}$. The maximum amidase activity occurred at a dilution rate of about $0.20 \mathrm{hr}^{-1}$ compared with about $0.30 \mathrm{hr}^{-1}$ for the wild type. At the higher dilution rates the amidase specific activity decreased much more slowly than did that of the wild type and was about 50 at a dilution rate of $0.50 \mathrm{hr}^{-1}$ when that of the wild type and fully constitutive strains had decreased to much lower values.

\section{Amidase activities of a semi-constitutive mutant}

Mutant $\mathrm{C}_{17} 7$ was isolated on $\mathrm{S} / \mathrm{F}$ medium after treatment of the wild-type strain by ultraviolet irradiation. It did not grow on $\mathrm{S} / \mathrm{L}$ plates and in batch culture in pyruvate medium was only slightly less sensitive than mutant $C_{\text {I I }}$ to catabolite repression by $10 \mathrm{mM}$-succinate. In the absence of inducer, mutant $\mathrm{C} I 7$ synthesized amidase at about half the differential rate of the fully induced wild type and could be fully induced by adding substrate or non-substrate inducers to the medium (Brammar et al. 1967). 
Figure 4 gives the results for amidase activity of mutant $\mathrm{C}_{17}$ in continuous culture on the succinate +acetamide medium. The general shape of the curve was very like that of the wild-type strain, but the sharp peak of maximum amidase activity occurred at a dilution rate of about $0.2 \mathrm{hr}^{-1}$. The amidase specific activity at low dilution rates was higher than that of the wild type, as might be expected from a partially constitutive mutant, and it is possible to consider the amidase curve for mutant $\mathrm{C} I 7$ as intermediate between that of the fully inducible wild type and the fully constitutive C I I. The rate of decrease in amidase specific activity at the higher dilution rates was about the same as that of the wild type. It was not possible to continue these measurements at dilution rates greater than $0.50 \mathrm{hr}^{-1}$ since this strain deteriorated at higher growth rates and tended to grow on the walls of the culture vessel.

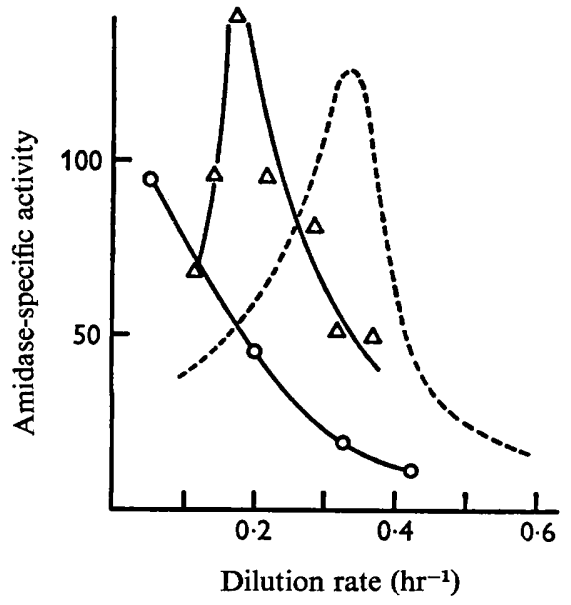

Fig. 4

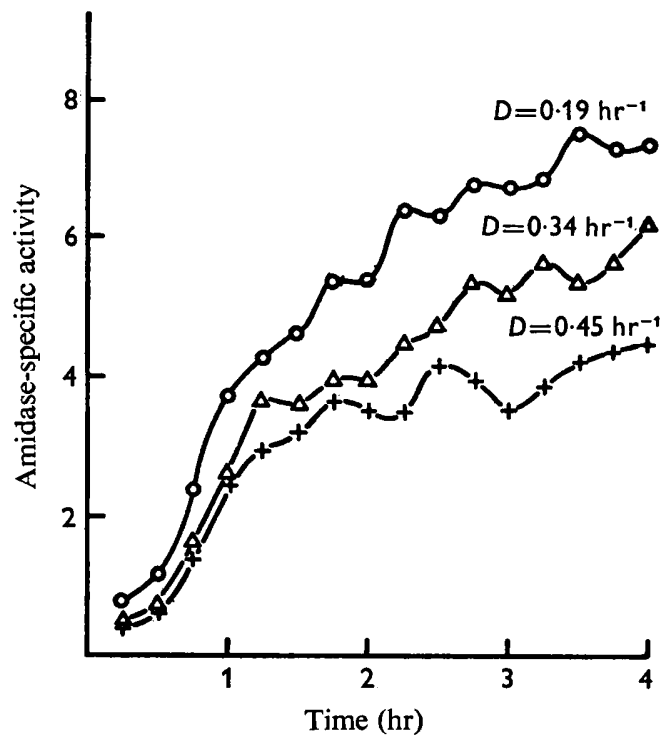

Fig. 5

Fig. 4. The amidase-specific activities of a magno-constitutive mutant $\mathrm{C}$ I I $(\mathrm{O}-\mathrm{O})$ and a semi-constitutive mutant $C I 7(\triangle-\triangle)$ grown in continuous culture under steady-state conditions in minimal medium containing ro mM-succinate $+20 \mathrm{~mm}$-acetamide. ---- , wild type on the same medium.

Fig. 5. Amidase synthesis by Pseudomonas aeruginosa 8602 wild type in continuous culture following a change of medium from minimal salts + Io mM-succinate to minimal salts + $10 \mathrm{~mm}$ $N$-acetylacetamide.

\section{Amidase induction by a non-substrate inducer in continuous culture}

The non-substrate amidase inducer $N$-acetylacetamide is not sufficiently stable to permit its use for prolonged studies in continuous culture, but we had previously found that it could be used for experiments of at least $4 \mathrm{hr}$ duration and we examined the synthesis of amidase by continuous cultures growing at different rates during the transition period when the ingoing medium was changed from $10 \mathrm{mM}$-succinate to 20 mM-succinate + Io mM- $N$-acetylacetamide (Boddy et al. 1967). The increase in succinate concentration allowed the bacteria to double in mass and during the transition period to the new steady state the effect of dilution rate on amidase induction 
could be studied. At higher dilution rates amidase induction during the transition period was severely repressed and there was a lag of several hours before the rate of synthesis became significant. Similar results were observed during the transition period from a medium containing Io mM-succinate to a medium containing Io mM-succinate + $20 \mathrm{~mm}$-acetamide, when the inducing amide was also available as a carbon source. We have now examined the rate of amidase synthesis by the wild type in continuous culture during the $4 \mathrm{hr}$ after the ingoing medium was changed from Io mM-succinate to Io mM-succinate + Io mM- $N$-acetylacetamide. Under these conditions there was no change in the growth rate and the bacterial mass remained the same. Figure 5 shows that at the three dilution rates tested there was only a short lag $(<0.5 \mathrm{hr})$ before amidase synthesis started. This corresponds to the building up of a sufficient inducer concentration in the culture vessel and there was no phase of severe repression as had been observed previously when the succinate concentration had been doubled at the time of adding the inducer. However, after $4 \mathrm{hr}$ the specific activities of the cultures at these three dilution rates were significantly different. Under these conditions the mean amidase specific activity of the culture growing at a dilution rate of $0.19 \mathrm{hr}^{-1}$ was greater than that at a dilution rate of $0.34 \mathrm{hr}^{-1}$ and almost twice that at $0.45 \mathrm{hr}^{-1}$, even though the $N$-acetylacetamide concentration was rising faster at the higher dilution rates.

\section{DISCUSSION}

Brammar \& Clarke (1964) showed that in exponentially growing cultures of Pseudomonas aeruginosa the substrate inducer acetamide could relieve catabolite repression of amidase synthesis by succinate. With carbon-starved resting bacteria it was possible to demonstrate that $N$-acetylacetamide, which is a much less effective inducer than acetamide, could also counteract succinate repression of amidase synthesis (Clarke \& Brammar, 1964). A similar interaction between induction and catabolite repression of $\beta$-galactosidase in Escherichia coli is now established (Clark \& Marr, 1964; Moses \& Prevost, 1966), although this was overlooked for a long time because the necessary quantitative experiments were not done.

We showed previously (Boddy et al. 1967) that in continuous culture the synthesis of Pseudomonas aeruginosa amidase was markedly affected by changes in the dilution rate of the culture and concluded that the extent of catabolite repression was related to the dilution rate. In studying the events during the transition period after changing the medium from succinate, to succinate + acetamide (doubling the concentration of carbon source), we found that at high dilution rates the synthesis of amidase was severely repressed. We suggested that the very high growth rate which occurred during the time when the culture was adapting to the new steady state resulted in a sufficiently high concentration of intermediary metabolites to prevent enzyme induction by acetamide. At the intermediate dilution rates of $0.30-0.50 \mathrm{hr}^{-1}$ the repression of enzyme synthesis was temporary and amidase synthesis started after a lag period. With the non-substrate inducer $N$-acetylacetamide this severe repression, with a lag in amidase synthesis, only occurred under conditions in which the concentration of the carbon source for growth was increased, and was not found in the present experiments, in which the ingoing medium was supplemented with $N$-acetylacetamide, leaving the succinate concentration unchanged. These results support the view that the severe catabolite repression observed during the transition period from one steady state to 
another is due to a temporary increase in the concentration of repressing intermediary metabolites. This metabolic adjustment during the transition period may be similar to the metabolic events occurring after the addition of glucose to an exponentially growing culture of Escherichia coli synthesizing $\beta$-galactosidase. Prevost \& Moses (1967) have shown that during transient repression of $\beta$-galactosidase synthesis by glucose there is a temporary increase in certain metabolites. The severe transient repression of $\beta$-galactosidase synthesis is relieved at the time the concentration of these metabolites falls to the original value.

We have shown now that the balance between induction and catabolite repression of an enzyme is also apparent in the regulation of the synthesis of an inducible enzyme in a culture growing under steady-state conditions. We interpret the curves obtained for the wild-type strain of Pseudomonas aeruginosa to mean that at low dilution rates catabolite repression is minimal and the rate of amidase synthesis is dependent mainly on the rate at which acetamide is presented to the bacteria. Thus, with increase in dilution rate the amidase specific activity under steady-state conditions increases. However, above $D=0.30 \mathrm{hr}^{-1}$ the growth rate has increased to the point where metabolic intermediates are being formed at a sufficiently high rate to cause significant catabolite repression. At higher dilution rates catabolite repression becomes dominant and at $D=0.60 \mathrm{hr}^{-1}$ the enzyme concentration has decreased considerably; but it must be realized that, even at these very high dilution rates, the specific activity of the culture is about the same as that of a batch culture grown overnight with acetamide.

In batch culture, the addition of a number of different carbon compounds to cultures induced by $\mathrm{N}$-acetylacetamide results in catabolite repression but succinate appears to be a better source of the actual repressing catabolite than is acetate. In continuous culture, the difference in the curves for the wild-type strain growing in acetamide, or succinate + acetamide, is probably due to the different rates of synthesis of metabolic intermediates from succinate and acetate respectively. This effect may be accentuated by the higher carbon-content of the succinate + acetamide medium used in these experiments. The interpretation of the curves obtained with the wild-type strain is supported by the results with mutants. Genetic experiments have shown that constitutivity markers ( $\mathrm{C}_{\mathrm{II}}, \mathrm{C}_{\mathrm{I}}$ ) ) are co-transduced with the amidase structural gene and we have concluded that a regulator gene is linked closely to the amidase structural gene (Brammar et al. 1967). The curves for the two fully constitutive mutants (C I , L 9) lack completely the part where, in the curves for the wild-type strain, we have suggested that induction is dominant. At low dilution rates the enzyme activities of mutant $C_{\text {I I }}$ approach the maximum value obtained with the wild type. Whereas the effect of catabolite repression is only noticed above $D=0.30 \mathrm{hr}^{-1}$ with the wild type, the effect is apparent at much lower dilution rates with mutants $C_{\text {II }}$ and $L$ 9. This implies that these particular mutations to constitutivity have also made the synthesis of amidase more susceptible to catabolite repression, presumably because the inducer is no longer able to counteract the repressing activity of the catabolite repressor molecule. The curve for the semi-constitutive mutant $\mathrm{C} I 7$ falls between the wild-type curve and the curve produced with the fully constitutive mutant $\mathrm{C}$ I I. The addition of inducer to this mutant increases the rate of amidase synthesis and might therefore also be expected to affect the catabolite repression.

The mutants with altered catabolite repressibility ( $\mathrm{L}_{9}, \mathrm{~L}_{\text {I I }}$ ) have not yet been analysed genetically. The genetic lesion in each case may be in the amidase regulator 
gene, or in a gene determining an enzyme concerned with succinate and acetate metabolism. The catabolite repressibility values, which were determined under standard conditions, could be correlated with the ability of the mutants to grow on S/L plates. Mutants with catabolite repressibility values of $80 \%$ or more grew no better than the wild type, but both mutant L I I, with catabolite repressibility value of $60 \%$, and mutant $\mathrm{L} 9$ ( $15 \%$ ) grew equally well on $\mathrm{S} / \mathrm{L}$ plates. These two mutants also gave curves for amidase activity in continuous culture which differed from those obtained for the corresponding wild-type and constitutive cultures at the high dilution rates where we have suggested that catabolite repression is dominant. The experiments with the wild type under steady-state conditions, with the substrate-inducer acetamide, showed that the maximum effective induction occurred at about $D=0.35 \mathrm{hr}^{-1}$. It was not possible to do extended steady-state experiments with the non-substrate inducer $N$-acetylacetamide since it is not sufficiently stable. In the experiments described in this paper the cultures were tending to reach a constant value for amidase activity after about $4 \mathrm{hr}$. The highest value for amidase activity was observed at the lowest dilution rate $\left(D=0.19 \mathrm{hr}^{-1}\right)$ and the values at 0.35 and $0.45 \mathrm{hr}^{-1}$ were significantly lower even though the $\mathrm{N}$-acetylacetamide concentration in the vessel increased more slowly at the low dilution rate. These results superficially resemble those obtained for constitutive cultures. In this case, however, the important factor is the difference in inducer activity of $\mathrm{N}$-acetylacetamide and acetamide. With carbon-starved bacteria, values obtained for $K_{\text {Ind }}$ were approximately I mM for $N$-acetylacetamide and $0.01 \mathrm{mM}$ for acetamide, (Brammar, 1965) and in batch-culture experiments acetamide was much more effective in relieving the effects of catabolite repression. In continuous culture, $\mathrm{N}$-acetylacetamide is too weak an inducer to allow any increase in the rate of amidase synthesis by the culture as the dilution rate is increased. With an increase in dilution rate from 0.19 to $0.45 \mathrm{hr}^{-1}$ only the increasing effect of catabolite repression with dilution rate was observed.

These results with continuous cultures confirm the observations made with batch cultures on the relationship between induction and repression of amidase synthesis in Pseudomonas aeruginosa 8602. The general conclusion is that the effect of an inducer will depend on the metabolic state of the organism with respect to the production of catabolite repressor molecules. When the internal concentration of the repressing metabolites is high the catabolite repression is severe and changes in inducer concentration have little effect on the rate of amidase synthesis. In a batch culture provided with acetamide and succinate as carbon sources this results in diauxic growth. At the other extreme, with a depleted carbon pool, catabolite repression is minimal and the rate of enzyme synthesis depends on the concentration of inducer and can be increased to the point at which the culture is fully induced. Between these two conditions, there is a partial repression of amidase synthesis by catabolic intermediates which may be increased or decreased by changes in the inducer concentration. The extent to which catabolite repression can be affected by changes in inducer concentration will depend on the relative effectiveness of the inducer and the repressing catabolites. Acetamide is more effective as an inducer than $N$-acetylacetamide, probably because it has a higher affinity for the inducer-binding site of its cytoplasmic repressor. The actual effector molecule is not yet known for catabolite repression in this system, but it is clear from these results that a competitive relationship exists between the inducer and the repressing catabolite. This competitive relationship can only readily be observed 
when the inducer is very active, and this probably requires it to have a high affinity for the inducer binding site of its specific cytoplasmic repressor. In continuous culture, variation of the growth substrates and changes in the growth rate of the culture may make it possible to examine in detail this balance of enzyme synthesis.

\section{REFERENCES}

Boddy, A., Clarke, P. H., Houldsworth, M. \& Lilly, M. D. (1967). Regulation of amidase synthesis by Pseudomonas aeruginosa 8602 in continuous culture. J. gen. Microbiol. 48, 137.

Brammar, W. J. (1965). The control of amidase synthesis in Pseudomonas aeruginosa by induction and repression mechanisms. Ph.D. thesis, University of London.

Brammar, W. J. \& Clarke, P. H. (I964). Induction and repression of Pseudomonas aeruginosa amidase. J. gen. Microbiol. 37, 307.

Brammar, W. J., Clarke, P. H. \& Skinner, A. J. (1967). Biochemical and genetic studies with regulator mutants of the Pseudomonas aeruginosa 8602 amidase system. J. gen. Microbiol, 47, 87.

Clark, D. J. \& MARr, A. G. (1964). Studies on the repression of $\beta$-galactosidase in Escherichia coli. Biochim. biophys. Acta 92, 85.

Clarke, P. H. \& Brammar, W. J. (1964). Regulation of bacterial enzyme synthesis by induction and repression. Nature, Lond. 203, 1153.

GoRINI, L. (1960). Antagonism between substrate and repressor in controlling the formation of a biosynthetic enzyme. Proc. natn. Acad. Sci. U.S.A. 46, 682.

Hamilton, W. A. \& Dawes, E. A. (1959). A diauxic effect with Pseudomonas aeruginosa. Biochem. J. $71,25 P$.

Hamlin, B. T., NG, F. M.-W. \& Dawes, E. A. (1967). Regulation of enzymes of glucose metabolism in Pseudomonas aeruginosa by citrate. In Microbial Physiology and Continuous Culture. Ed. by E. O. Powell, C. G. T. Evans, R. E. Strange \& D. W. Tempest, p. 2 I I. London: H.M.S.O.

Kelly, M. \& Clarke, P. H. (1962). An inducible amidase produced by a strain of Pseudomonas aeruginosa. J. gen. Microbiol. 27, 305.

MaGaSaniK, B. (1961). Catabolite repression. Cold Spring Harb. Symp. quant. Biol. 26, 249.

McFall, E. \& MANDELSTAM, J. (1963). Specific metabolic repression of three induced enzymes in Escherichia coli. Biochem. J. 89, 391.

Moses, V. \& Prevost, C. (1966). Catabolite repression of $\beta$-galactosidase synthesis in Escherichia coli. Biochem. J. roo, 336.

NG, F. M.-W. \& DAWES, E. A. (1967). Regulation of enzymes of glucose metabolism by citrate in Pseudomonas aeruginosa. Biochem. J. 104, $48 \mathrm{P}$.

Prevost, C. \& Moses, V. (1967). Pool sizes of metabolic intermediates and their relation to glucose repression of $\beta$-galactosidase synthesis in Escherichia coli. Biochem. J. 103, 347. 